\title{
The Prognostic Effects of Temporal Muscle Thickness and Inflammatory-Nutritional Parameters on Survival in Lung Cancer Patients with Brain Metastasis
}

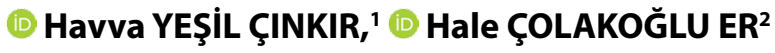 \\ 'Department of Medical Oncology, Gaziantep University Faculty of Medicine, Gaziantep-Turkey \\ ${ }^{2}$ Department of Radiology, Gaziantep University Faculty of Medicine, Gaziantep-Turkey
}

\begin{abstract}
OBJECTIVE
Brain metastasis develops in one-third of the patients with adult cancer and it causes significant morbidity and mortality on the patients. In this study, we aimed to show the prognostic effects of temporal muscle thickness and inflammatory (neutrophil-lymphocyte ratio, platelet-lymphocyte ratio and systemic inflammation response index) and prognostic nutritional index on survival in lung cancer patients with brain metastasis.
\end{abstract}

\section{METHODS}

In this study, a total of 94 cancer patients diagnosed with brain metastasis lung cancer were evaluated retrospectively. Routine blood sampling was tested within 10 days of brain MR imaging before the use of steroid therapy.

\section{RESULTS}

Temporal muscle thickness was significantly associated with overall survival, according to cut-off 4.32 ( $95 \% \mathrm{CI} 0.66-0.99 ; \mathrm{p}=0.040$ ). The risk of death increased by $18.8 \%$, with every millimeter reduction in temporal muscle thickness. Neutrophil-lymphocyte ratio, systemic inflammation response index and prognostic nutritional index were associated with overall survival $(\mathrm{p}=0.002, \mathrm{p}=0.008, \mathrm{p}=0.001$, respectively).

\section{CONCLUSION}

The findings showed that the use of temporal muscle thickness and inflammatory-nutritional parameters for the evaluation of sarcopenia were practical and rapid methods. In this group of patients with a short duration of survival, the prognosis of patients can be predicted, and their exposure to toxic treatment may be prevented by the evaluation of sarcopenia.

Keywords: Inflammation; lung cancer; sarcopenia; temporal muscle thickness.

Copyright $\odot$ 2020, Turkish Society for Radiation Oncology

\section{Introduction}

Brain metastasis develops in one-third of the patients with adult cancer. It causes significant morbidity and mortality on the patients.[1]
The primary cancer region is known in the majority of brain metastasis, while the primary region is not known in $5-10 \%$ of the patients. Most of the brain metastases arise from lung (40-50\%), breast (15-25\%), melanoma (5-20\%) and kidney (5-10\%).[2] 
Treatment options are surgical resection, radiotherapy (whole-brain radiotherapy or stereotactic radiosurgery) and systemic drugs. These treatments are applied according to the tumor location, number and size of the tumor in the brain, extracranial disease status, patient's performance status, histological and molecular characteristics of the tumor.[1]

Several prognostic factors have been identified for predicting the outcome of the treatments, which included the number of brain metastases, the extracranial tumor status and the Karnofsky performance scale. However, these factors were insufficient to predict survival.[3]

Cancer cachexia is characterized by systemic inflammation, negative protein and energy balance, loss of lean tissue mass.[4] Sarcopenia is defined as the mass and function of the skeletal muscle and has a core role in cancer-related cachexia.[4] It is associated with functional impairment and disability and can be used as an objective, measurable frailty parameter. Recent studies with various cancers have shown a decrease in long-term results with sarcopenia. [5,6] The majority of these observations were based on measurements of skeletal muscle index at the level of the third lumbar spine obtained by computed tomography (CT) scans. In one study, there was a positive relationship between the psoas major muscle cross-sectional area and temporal muscle thickness (TMT).[7] Therefore, the measurement of craniofacial muscles concerning sarcopenia in patients with brain metastasis appears to be a suitable option.

Previous studies have shown the relationship between temporal muscle thickness measurement and survival in lung, breast and malignant melanoma patients with brain metastasis. $[8,9]$

Recently, it has been reported that the immunological status, including the state of inflammation and nutritional status, is important for survival in cancer. Routine immune and inflammatory cells, such as neutrophils, monocytes and lymphocytes in the systemic circulation, may contribute to tumor cell invasion and metastasis.[10] Different inflammation-based scores have been proposed and are considered useful in this respect.[11] Previous studies showed neutrophil-lymphocyte ratio (NLR) and platelet-lymphocyte ratio (PLR) as an indicator of inflammation; prognostic nutritional index (PNI) was also studied in patients with lung cancer for nutritional status.[12,13] Systemic inflammation response index (SIRI), another marker of inflammation, has been shown to be associated with prognosis in other cancers $[14,15]$ but has not previously been studied in lung cancer.
In this study, we aimed to show the prognostic impact of temporal muscle thickness (TMT) measured in brain MR images and inflammatory (NLR, PLR, SIRI) and nutritional parameter (PNI) on survival in lung cancer patients with brain metastasis.

\section{Materials and Methods}

\section{Patients and Study Design}

A total of 94 cancer patients diagnosed with brain metastasis lung cancer were evaluated at the Gaziantep University Faculty of Medicine between 2011-2018, retrospectively. This study was approved by the ethics committee of Gaziantep University. The inclusion criteria were as follows: available MR images examination of the brain, absence of craniectomy defect and muscle edema in the temporal muscle region, accessible laboratory results, confirmed pathologically as the small cell lung carcinoma (SCLC) and non-small cell lung carcinoma (NSCLC). In SCLC, patients who had brain metastasis at the time of diagnosis and who had not received radiotherapy before were included in this study. Informed consent was obtained from all individual participants before brain MR imaging. Routine blood sampling, such as the counts of neutrophils, lymphocytes, monocytes, platelets and albumin, was tested within 10 days of brain MR imaging before the use of steroid therapy. Systemic inflammation and nutrition parameters were calculated according to the following formula:

NLR: Peripheral neutrophil count $\left(\right.$ per $\left.\mathrm{mm}^{3}\right) / \mathrm{lym}$ phocyte count ratio (per $\mathrm{mm}^{3}$ )

PLR: Peripheral platelet count $\left(\right.$ per $\left.\mathrm{mm}^{3}\right) /$ lymphocyte count ratio (per $\mathrm{mm}^{3}$ )

SIRI: Peripheral neutrophil count $\left(\right.$ per $\left.\mathrm{mm}^{3}\right) \mathrm{x}$ monocyte count (per $\left.\mathrm{mm}^{3}\right) /$ lymphocyte count (per $\mathrm{mm}^{3}$ )

PNI: [10 $\times$ serum albumin $(\mathrm{g} / \mathrm{dL})]+[0.005 \times$ total lymphocyte count $\left(\right.$ per $\left.\mathrm{mm}^{3}\right)$ ]

\section{Analyses of the Temporal Muscle Thickness}

Temporal muscle thickness (TMT) measurement at diagnosis of brain metastasis was measured on the contrast-enhanced axial T1- weighted MR images. Measurement was made perpendicular to the long axis of the temporal muscle at the level of the orbital roof. On axial T1- weighted MR images, left and right TMT were measured by an experienced radiologist for all patients. The radiologist was blinded to the results of patients' clinical characteristics. The parameters of axial T1- weighted MR images were a TR of $160 \mathrm{~ms}$, a TE of $2 \mathrm{~ms}$, a FOV of $210 \mathrm{~mm}$, a slice thickness of $5 \mathrm{~mm}$, 
a gap of $1 \mathrm{~mm}$. Examples of TMT assessment on MR images was shown in Figure 1.

\section{Statistical Analysis}

Median TMT was assessed by summing the measurements from each side and then dividing by two, which resulted in one variable- the median TMT per patient. TMT was grouped according to the median per group. Overall survival (OS) was defined as from the diagnosis of brain metastasis to death or date of the last follow-up. The survival durations were presented as weeks. The Kaplan-Meier was applied to retrieve survival curves. The log-rank test was used to investigate differences between groups. TMT was entered as a scale variable in a Cox regression model to investigate the association with survival times. The strength of the association between two scale variables was calculated using a Spearman correlation coefficient. A two-tailed p-value of $<0.05$ was considered statistically significant. Statistical analyses were performed using the Statistical Package for the Social Sciences, Version 22.0 (SPSS, Chicago, Illinois).

\section{Results}

The patients' characteristics are shown in Table 1. Overall, 94 patients were available for further analysis,

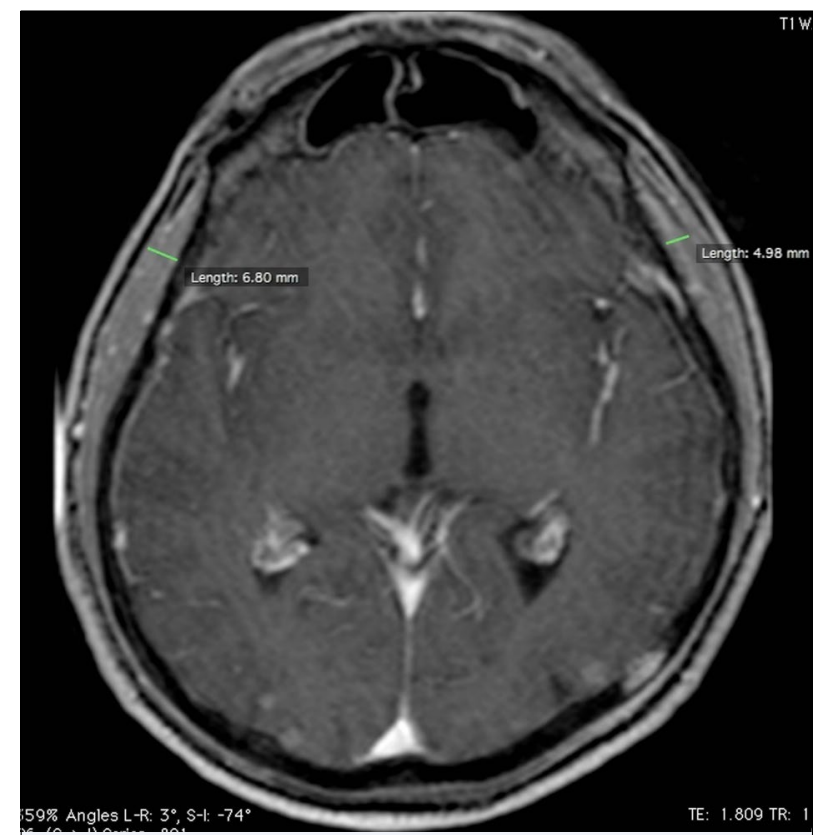

Fig. 1. Temporal muscle thickness measurement on a 52 years- old-male patient with non-small cell lung cancer. Contrast-enhanced axial T1 weighted MR image shows temporal muscle thickness measurement bilaterally. assembled from two groups that consisted of $71 \mathrm{pa}-$ tients with brain metastases from NSCLC and 23 patients with brain metastases from SCLC. The median age was 60 (range: $39-78$ ); 87.2\% of the patients were male. In the NSCLC group, the majority of patients (50; 70.4\%) had adenocarcinoma, while 21 (29.5\%) patients had squamous cell carcinoma. The median TMT on the right side was $4.43 \mathrm{~mm}$ (range 1.9-9.5), and $4.36 \mathrm{~mm}$ (range 1.9-8.4) on the left side, resulting in a median of $4.38 \mathrm{~mm}$ (range 1.9-8.7) in the NSCLC group. The median TMT on the right side was $4.22 \mathrm{~mm}$ (range 2.4-7.7), and $3.98 \mathrm{~mm}$ (range 2.2-6.2) on the left side, resulting in a median of $3.90 \mathrm{~mm}$ (range 2.4-6.7) in the SCLC group. According to median TMT 4.32, no difference was observed between the groups of metastasis at the time of diagnosis $(\mathrm{p}=0.778)$, brain metastasis at the time of diagnosis $(p=0.148)$ and the number of additional metastatic regions ( $\mathrm{p}=0.548$ ) (Chi-square test). Survival analysis was performed according to median TMT 4.32 value and Table 2 showed survival values for TMT and inflammatory-nutritional parameters. The OS analysis of all lung cancer patients was 13 weeks, according to subgroups of TMT, 12 weeks for TMT $<4.32$ and 15 weeks for $\geq 4.32$. There was no difference in OS between the values of $<4.32$ and $\geq 4.32$ in the NSCLC group (15 weeks). In the SCLC group, the OS was 19 weeks for $\geq 4.32$, six weeks for $<4.32$ ( $\mathrm{p}=0.016$ ) (Fig. 2).

The cut-off point for NLR from inflammatory parameters was 3.80. Survival was 33 weeks for NLR $<3.80$, while six weeks for $\geq 3.80(\mathrm{p}=0.002) ; 16$ weeks for $P L R<188,10$ weeks for $\geq 188$ ( $\mathrm{p}=0.125$ ). The cut-off point for SIRI was 2.81. Median OS analysis was seven weeks for SIRI $<2.81$ and 29 weeks for $\geq 2.81$ ( $p=0.008$ ). According to the PNI parameter for nutritional assess-

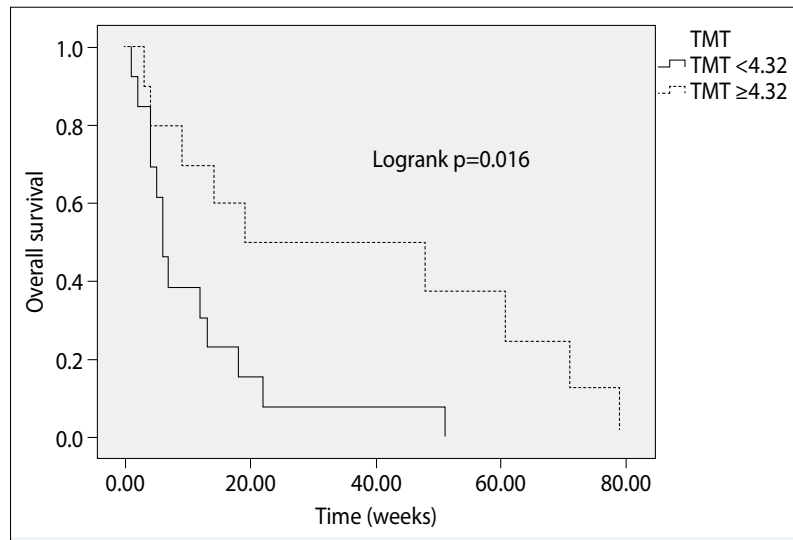

Fig. 2. The Overall survival analysis of small cell lung cancer patients between the median temporal muscle thickness values of $<4.32$ and $\geq 4.32$. 
Table 1 An overview of the patients' characteristics

\begin{tabular}{|c|c|c|}
\hline & $\begin{array}{c}\operatorname{SCLC}(n=23) \\
n(\%)\end{array}$ & $\begin{array}{c}\operatorname{NSCLC~}(n=71) \\
n(\%)\end{array}$ \\
\hline \multicolumn{3}{|l|}{ Gender } \\
\hline Female & $3(3.1)$ & $9(9.5)$ \\
\hline Male & $20(21.2)$ & $62(65.9)$ \\
\hline \multicolumn{3}{|l|}{ Pathologic subtype } \\
\hline Adeno cancer & & $50(70.4)$ \\
\hline Squamoz cancer & & $21(29.5)$ \\
\hline \multicolumn{3}{|c|}{ Metastatic at the time of diagnosis } \\
\hline Yes & $23(100)$ & $59(83.0)$ \\
\hline No & 0 & $12(16.9)$ \\
\hline \multicolumn{3}{|c|}{ Brain metastatic at the time of diagnosis } \\
\hline Yes & $12(52.1)$ & $37(52.1)$ \\
\hline No & $11(47.8)$ & $34(47.8)$ \\
\hline \multicolumn{3}{|c|}{ Additional metastasis site } \\
\hline Absent & $2(8.6)$ & $10(14)$ \\
\hline 1 site & $4(17.3)$ & $18(25.3)$ \\
\hline 2 site & $9(39.1)$ & $33(46.4)$ \\
\hline$\geq 3$ site & $8(34.7)$ & $10(14)$ \\
\hline \multicolumn{3}{|l|}{ Status } \\
\hline Alive & $1(4.3)$ & $18(25.3)$ \\
\hline \multirow[t]{3}{*}{ Dead } & $22(95.6)$ & $53(74.6)$ \\
\hline & Mean士SD & Mean \pm SD \\
\hline & Median (range) & Median (range) \\
\hline \multirow[t]{2}{*}{ TMT-Right site } & $4.45 \pm 1.32$ & $4.52 \pm 1.49$ \\
\hline & $4.22(2.4-7.7)$ & $4.43(1.9-9.5)$ \\
\hline \multirow[t]{2}{*}{ TMT-Left site } & $4.22 \pm 1.11$ & $4.44 \pm 1.26$ \\
\hline & $3.98(2.2-6.2)$ & $4.36(1.9-8.4)$ \\
\hline \multirow[t]{2}{*}{ TMT-Median } & $4.33 \pm 1.12$ & $4.48 \pm 1.31$ \\
\hline & $3.90(2.4-6.7)$ & $4.38(1.9-8.7)$ \\
\hline \multirow[t]{2}{*}{ Neutrophil $\left(\mathrm{mm}^{3}\right)$} & $5.452 \pm 2.136$ & $7.492 \pm 3.466$ \\
\hline & $5.320(1.450-10.170)$ & $6.670(1.980-16.720)$ \\
\hline \multirow[t]{2}{*}{ Lymhocyte $\left(\mathrm{mm}^{3}\right)$} & $1.618 \pm 0.659$ & $1.639 \pm 0.869$ \\
\hline & $1.500(0.530-3.150)$ & $1.490(0.390-4.560)$ \\
\hline \multirow[t]{2}{*}{ Monocyte $\left(\mathrm{mm}^{3}\right)$} & $762 \pm 285$ & $0.883 \pm 0.415$ \\
\hline & $0.680(0.260-1.460)$ & $0.800(0.80-2.090)$ \\
\hline \multirow{2}{*}{ Platelet $\left(\mathrm{mm}^{3}\right)$} & $326 \pm 120$ & $295 \pm 116$ \\
\hline & $334(88-577)$ & $293(35-603)$ \\
\hline
\end{tabular}

SCLC: Small cell lung cancer; NSCLC: Non-small cell lung cancer; TMT: Temporal muscle thickness

ment, survival was 33 weeks for $\geq 42.7$ and $<42.7$ for six weeks $(\mathrm{p}=0.001)$. Median OS in the SCLC group was six weeks for TMT $<4.32$ and 19 weeks for $\geq 4.32$ $(\mathrm{p}=0.016)$. OS was 19 weeks for NLR $<3.80$, five weeks for $\geq 3.80$ ( $\mathrm{p}=0.001)$; 18 weeks for PLR $<188$, five weeks for $\geq 188$ ( $\mathrm{p}=0.106)$; nine weeks for $\operatorname{SIRI}<2.81,12$ weeks for $\geq 2.81$ ( $\mathrm{p}=0.405)$; 22 weeks for $\mathrm{PNI} \geq 42.7$, four weeks for $<42.7(\mathrm{p}=0.000)$. According to the Spearman correlation analysis, there was no correlation between median TMT and NLR, PLR, SIRI and PNI.
A survival analysis using a Cox regression model was performed with baseline TMT diameters to predict survival time in general lung cancer patients, SCLC and NSCLC group. In general group, according to cut-off 4.32, TMT was statistically significantly associated with survival prognosis with an HR of 0.812 (95\% CI 0.660.99; $\mathrm{p}=0.040$ ). Markedly, the risk of death increased by $18.8 \%$ with every millimeter reduction in TMT. However, this survival difference was not detected in SCLC and NSCLC subgroups ( $\mathrm{p}=0.059 ; \mathrm{p}=0.124)$. 
Table 2 Survival values for the temporal muscle thickness and inflammatory-nutritional parameters

\begin{tabular}{ccccc} 
& $\begin{array}{c}\text { SCLC } \\
\text { Weeks }\end{array}$ & $\mathbf{p}$ & $\begin{array}{c}\text { NSCLC } \\
\text { Weeks }\end{array}$ & $\mathbf{p}$ \\
\hline $\begin{array}{c}\text { TMT median } \\
<4.32\end{array}$ & 6 & 0.016 & & 0.556 \\
$\geq 4.32$ & 19 & & 15 & \\
NLR & & 0.001 & 15 & \\
$\quad<3.80$ & 19 & & 33 & 0.002 \\
$\geq 3.80$ & 5 & & 6 & \\
PLR & & 0.106 & & 0.125 \\
$<188$ & 18 & & 16 & \\
$\geq 188$ & 5 & & 10 & \\
SIRI & & 0.405 & & 0.008 \\
$\quad<2.81$ & 9 & & 29 & \\
$\geq 2.81$ & 12 & & 7 & \\
PNI & & $<0.001$ & & 0.001 \\
$\quad<42.7$ & 4 & & 6 & \\
$\geq 42.7$ & 22 & & 36 &
\end{tabular}

TMT: Temporal muscle thickness; NLR: Neutrophil-lymphocyte ratio; PLR: Platelet-lymphocyte ratio; SIRI: Systemic inflammation response index PNI: Prognostic nutritional index; Statistically significant values are in italics $(\mathrm{p}<0.05)$

In the multivariate cox analysis, including TMT, NLR, PLR, SIRI and PNI parameters, only PNI showed a statistically significant association with survival prognosis (HR 0.483; 95\% CI 0.28-0.82; $\mathrm{p}=0.008$ ).

\section{Discussion}

According to our experience in the daily population, an increase in the incidence of brain metastases is observed upon the prolongation of the survival of cancer patients and the progression of imaging techniques.

$\mathrm{BM}$ is a heterogeneous group with the varied response to treatment and survival. Therefore, it is important to consider the various factors affecting the prognosis of patients with BM before therapeutic decision making.

In this study, the effects of both the measurement of TMT and inflammatory and nutritional parameters on survival in brain metastatic lung adenocarcinoma were investigated.

Multiple factors play a role in the etiology of cancerassociated cachexia, which are malnutrition, catabolic metabolism, chronic inflammation, paraneoplastic effects, anti-neoplastic therapies, psychological reasons (such as anxiety disorder and depression) and other factors.

Sarcopenia is a loss of muscle and is used as an indicator of cachexia. In previous publications, craniofacial skeletal muscles have been shown to be beneficial in predicting muscle mass loss, in addition to abdominal skeletal muscles. In Leitner et al's study, a high correlation was found between TMT and lumbar skeletal muscle measurements.[16] In the study of Swartz et al., paravertebral muscles and sternocleidomastoid muscles in the third cervical vertebra region were measured in patients with head and neck cancer.[17] These results were correlated with muscle measurements in the third lumbar vertebra region. Kilgour et al. showed a loss of muscle mass with the cross-sectional measurement of neck muscles in the second cervical vertebrae in elderly patients.[18] Lisiecki et al.s study, TMT was measured in patients with mandibular fractures due to trauma. [19] As a result of the study, the duration of stay in the hospital, intensive care unit and the ventilator was reported longer in patients with low TMT. In a study with children diagnosed with non-syndromic craniosynostosis, a negative correlation was found between temporal muscle volume and length of hospital stay.[20]

In our study, there was a significant relationship between TMT and survival. The overall survival of patients with brain metastatic lung cancer was 13 weeks. In these patients, the risk of death increased by $18.8 \%$ with every millimeter reduction in TMT. The patients were classified into two main groups. Unfortunately, there was no difference in survival according to median TMT in SCLC and NSCLC group. This situation was thought to be due to the small number of patients in groups.

In the study of Further et al., median TMT was calculated as $5.9 \mathrm{~mm}$ in patients with NSCLC and survival difference was shown.[9] In the study of Leitner et al., the mean TMT value was $6.1 \mathrm{~mm}$.[16] In our study, the median TMT value of our patients was lower than the literature. This situation suggested that sarcopenia was advanced in our patients or there was a possibility of social difference.

In our study, the TMT value was not affected by age and gender. Further et al. reported a negative correlation between age and TMT.[9] They emphasized that physical condition was more important than the patient's age.

It was thought that the prognosis might be worse in patients with metastatic phase at the time of diagnosis, brain metastasis at the time of diagnosis or additional metastatic region other than brain metastasis. Chisquare analysis performed with this thought; however, there was no correlation between TMT and these clinical indicators ( $\mathrm{p}=0.778 ; \mathrm{p}=0.148 ; \mathrm{p}=0.548$ ).

Inflammation is recognized as one of the hallmarks of cancer.[21] Cancer-related inflammation plays an 
important role in the development and progression of cancer. Immune and inflammatory cells are considered to be essential components of the tumor microenvironment.[14]

The actual mechanisms underlying how the NLR, PLR and SIRI affect prognosis in patients with cancer are unclear. High NLR represents increased neutrophil count and/or decreased lymphocyte count; similarly, high PLR indicates increased platelet and/or decreased lymphocyte count. In preclinical studies, neutrophils have been shown to increase cancer cell metastasis, invasion and proliferation, but also help to escape the immune system and thus promote tumor activity. Platelets can collect granulocytes and facilitate the early formation of metastatic niches. Platelet-derived growth factors and direct contact of the tumor cell with platelet can activate tumor pathways in malignant cells and facilitate the formation of metastases. Lymphocytes have strong effects that can inhibit tumor cell migration and growth and stimulate cytotoxic cell death. Monocytes can induce tumor cells or immune cells by producing chemokines, such as vascular endothelial growth factor, tumor necrosis factor-alpha and monocyte chemoattractant protein-1, to promote tumor formation, angiogenesis and distant metastasis.[22] Yu et al. conducted a meta-analysis of a large sample with 7219 patients and demonstrated that a high NLR predicted poor OS (HR: 1.46; 95\% CI: 1.30-1.64).[23] Liu et al. found that a high PLR was a significant independent prognostic factor for patients with NSCLC (HR: 2.025; 95\% CI: 1.405-2.919; p<0.001).[24] Dong et al. reported that NLR was an independent prognostic factor for poor OS (HR: 1.954; 95\% CI: 1.172-3.257; $\mathrm{p}=0.01$ ), but not the PLR.[25] Mitsuya et al. reported a survival benefit in patients with low NLR levels who had been operated for brain metastasis (14 months versus five months, $\mathrm{p}=0.01$ ).[26] Doi et al. reported that low NLR levels and positive programmed death-1 (PD-1) values in patients with brain metastasis might be associated with a strong immune response to tumor.[27]

Lung cancer is a heterogeneous group and has different subtypes. Therefore, Igor et al. investigated that NLR and PLR parameters may differ between subtypes in lung cancer patients.[28] As a result of their study, there was no difference between these subtypes concerning NLR and PLR parameters. Similar results were found in the SCLC and NSCLC groups for NLR and PLR in our study (for NLR $\mathrm{p}=0.001, \mathrm{p}=0.002$; for PLR $\mathrm{p}=0.106, \mathrm{p}=0.125$ ).

The SIRI parameter has been studied in various cancers and can be used as a prognostic marker.[14,15,29]
According to the literature review, SIRI has not been studied previously in lung cancer.

In our study, low SIRI was associated with improved survival in the analysis of all lung cancer patients (HR: 1.70; 95\% CI: 1.07-2.71; $\mathrm{p}=0.023$ ). In subgroup analysis, the survival of SIRI was statistically significant in NSCLC and no difference was observed in SCLC $(p=0.008 ; p=0.405)$. In this case, the small number of patients in the SCLC group was thought to be the causative factor.

$\mathrm{PNI}$ is a parameter that shows the immune and nutritional status calculated by the combination of serum albumin and lymphocyte values. It predicts the prognosis of patients with cancer independent of origin. [30] It has not been studied previously in patients with brain metastasis. In our study, high PNI values were associated with increased survival in all lung cancer patients (HR: 2.59; 95\% CI: 1.61-4.15; p<0.001). In subgroup analysis, survival in both SCLC and NSCLC was statistically significant $(\mathrm{p}<0.001, \mathrm{p}=0.001)$.

In patients with brain metastases, temporal muscle measurements were performed to evaluate sarcopenia more quickly and easily. In one study, TMT measurement was shown to last 30 seconds. [8] Similarly, our measurements were made in a short time. Abdominal imaging was not performed during the diagnosis of brain metastasis in the majority of patients. Therefore, abdominal muscular thickness measurement was not used for the evaluation of sarcopenia. Because of this reason, TMT measurement is useful for the evaluation of sarcopenia in patients with brain metastasis. The disadvantage of TMT measurement is that it is affected by oral and dental diseases and rheumatic diseases (e.g. temporal arteritis) in the differential diagnosis of headache in elderly patients. Therefore, both sides of the muscle were measured and analyzed according to the median value.

Patients who had steroid use and infection because of the effect of steroid use on hematological parameters and hypoalbuminemia as a negative acute-phase reaction during infection were not included in this study.

There were restrictive aspects of our study. First, the number of patients in this study was low, and the number of patients in the subgroups was not evenly distributed; the second limitation was that this was a retrospective study.

The TMT value of our patients was lower than the other studies. The reason for this was the inclusion of more complicated cases in our study because our center was the tertiary center. It is thought that the importance of TMT and inflammatory-nutritional status assessment will be better understood in the future by 
studies that are prospectively designed and included a larger number of patients.

\section{Conclusion}

In this study, the findings suggest the use of TMT and inflammatory-nutritional parameters for the evaluation of sarcopenia were practical and rapid methods in lung cancer patients with brain metastasis. In this group of patients with a short duration of survival, the prognosis of patients can be predicted and their exposure to toxic treatment may be prevented by the evaluation of sarcopenia.

Peer-review: Externally peer-reviewed.

Conflict of Interest: The authors declare that they have no conflict of interest.

Ethics Committee Approval: The protocol and informed consent documentation were reviewed and approved by the Independent Ethics Committee of the University and agreed with the ethical principles of the Declaration of Helsinki.

Financial Support: Financial and material support was not recevied.

Authorship contributions: Concept - H.Y.Ç., H.Ç.E.; Design - H.Y.Ç., H.Ç.E.; Supervision - H.Y.Ç., H.Ç.E.; Funding - H.Y.Ç.; Materials - H.Y.Ç., H.Ç.E.; Data collection and/or processing - H.Y.Ç., H.Ç.E.; Data analysis and/or interpretation - H.Y.Ç., H.Ç.E.; Literature search - H.Y.Ç.; Writing - H.Y.Ç.; Critical review - H.Y.Ç., H.Ç.E.

\section{References}

1. Arvold ND, Lee EQ, Mehta MP, Margolin K, Alexander BM, Lin NU, et al. Updates in the management of brain metastases. Neuro Oncol 2016;18(8):1043-65.

2. Ekici K, Temelli O, Dikilitas M, Halil Dursun I, Bozdag Kaplan N, Kekilli E. Survival and prognostic factors in patients with brain metastasis: Single center experience. J BUON 2016;21(4):958-63.

3. Kondziolka D, Parry PV, Lunsford LD, Kano H, Flickinger JC, Rakfal S, et al. The accuracy of predicting survival in individual patients with cancer. J Neurosurg 2014;120(1):24-30.

4. Fearon K, Strasser F, Anker SD, Bosaeus I, Bruera E, Fainsinger RL, et al. Definition and classification of cancer cachexia: an international consensus. Lancet Oncol 2011;12(5):489-95.

5. Prado CM, Lieffers JR, McCargar LJ, Reiman T, Sawyer MB, Martin L, et al. Prevalence and clinical implications of sarcopenic obesity in patients with solid tumours of the respiratory and gastrointesti- nal tracts: a population-based study. Lancet Oncol 2008;9(7):629-35.

6. Tamandl D, Paireder M, Asari R, Baltzer PA, Schoppmann SF, Ba-Ssalamah A. Markers of sarcopenia quantified by computed tomography predict adverse longterm outcome in patients with resected oesophageal or gastro-oesophageal junction cancer. Eur Radiol 2016;26(5):1359-67.

7. Ranganathan K, Terjimanian M, Lisiecki J, Rinkinen J, Mukkamala A, Brownley C, et al. Temporalis muscle morphomics: the psoas of the craniofacial skeleton. J Surg Res 2014;186(1):246-52.

8. Furtner J, Berghoff AS, Albtoush OM, Woitek R, Asenbaum U, Prayer D, et al. Survival prediction using temporal muscle thickness measurements on cranial magnetic resonance images in patients with newly diagnosed brain metastases. Eur Radiol 2017;27(8):3167-73.

9. Furtner J, Berghoff AS, Schöpf V, Reumann R, Pascher $\mathrm{B}$, Woitek R, et al. Temporal muscle thickness is an independent prognostic marker in melanoma patients with newly diagnosed brain metastases. J Neurooncol 2018;140(1):173-8.

10. Diakos CI, Charles KA, McMillan DC, Clarke SJ. Cancer related inflammation and treatment effectiveness. Lancet Oncol 2014;15(11):e493-503.

11. Chen J, Fang A, Chen M, Tuoheti Y, Zhou Z, Xu L, et al. A novel inflammation-based nomogram system to predict survival of patients with hepatocellular carcinoma. Cancer Med 2018;7(10):5027-35.

12. Sun H, Hu P, Du J, Wang X. Predictive value of inflammatory indexes on the chemotherapeutic response in patients with unresectable lung cancer: A retrospective study. Oncol Lett 2018;15(3):4017-25.

13. Shimizu K, Okita R, Saisho S, Yukawa T, Maeda A, Nojima Y, et al. Prognostic nutritional index before adjuvant chemotherapy predicts chemotherapy compliance and survival among patients with non-small-cell lung cancer. Ther Clin Risk Manag 2015;11:1555-61.

14. Xu L, Yu S, Zhuang L, Wang P, Shen Y, Lin J, et al. Systemic inflammation response index (SIRI) predicts prognosis in hepatocellular carcinoma patients. Oncotarget 2017;8(21):34954-60.

15. Qi Q, Zhuang L, Shen Y, Geng Y, Yu S, Chen H, et al. A novel systemic inflammation response index (SIRI) for predicting the survival of patients with pancreatic cancer after chemotherapy. Cancer 2016;122(14):2158-67.

16. Leitner J, Pelster S, Schöpf V, Berghoff AS, Woitek R, Asenbaum U, et al. High correlation of temporal muscle thickness with lumbar skeletal muscle crosssectional area in patients with brain metastases. PLoS One 2018;13(11):e0207849.

17. Swartz JE, Pothen AJ, Wegner I, Smid EJ, Swart KM, de Bree R, et al. Feasibility of using head and neck CT 
imaging to assess skeletal muscle mass in head and neck cancer patients. Oral Oncol 2016;62:28-33.

18. Kilgour AH, Subedi D, Gray CD, Deary IJ, Lawrie SM, Wardlaw JM, et al. Design and validation of a novel method to measure cross-sectional area of neck muscles included during routine MR brain volume imaging. PLoS One 2012;7(4):e34444.

19. Lisiecki J, Zhang P, Wang L, Rinkinen J, De La Rosa S, Enchakalody B, et al. Morphomic measurement of the temporalis muscle and zygomatic bone as novel predictors of hospital-based clinical outcomes in patients with mandible fracture. J Craniofac Surg 2013;24(5):1577-81.

20. Rinkinen J, Zhang P, Wang L, Enchakalody B, Terjimanian M, Holcomb S, et al. Novel temporalis muscle and fat pad morphomic analyses aids preoperative risk evaluation and outcome assessment in nonsyndromic craniosynostosis. J Craniofac Surg 2013;24(1):250-5.

21. Hanahan D, Weinberg RA. Hallmarks of cancer: the next generation. Cell 2011;144(5):646-74.

22. Chen Y, Wang W, Zhang X, Yu X, Xi K, Wen Y, et al. Prognostic significance of combined preoperative platelet-to-lymphocyte ratio and lymphocytetomonocyte ratio in patients undergoing surgery with stage IB non-small-cell lung cancer. Cancer Manag Res 2018;10:5411-22.

23. Yu Y, Qian L, Cui J. Value of neutrophil-to-lymphocyte ratio for predicting lung cancer prognosis: a meta-analysis of 7,219 patients. Mol Clin Oncol 2017;7(3):498-506.

24. Liu H, Wu Y, Wang Z, Yao Y, Chen F, Zhang H, et al. Pretreatment platelet-to-lymphocyte ratio (PLR) as a predictor of response to first-line platinum-based chemotherapy and prognosis for patients with non-small cell lung cancer. J Thorac Dis 2013;5(6):783-9.

25. Guo D, Li M, Chen D, Jing W, Zhu H, Fu L, et al. Neutrophil-to-lymphocyte ratio is superior to plateletto-lymphocyte ratio as a prognostic predictor in advanced non-small-cell lung cancer treated with first-line platinum-based chemotherapy. Future Oncol 2019;15:625-35.

26. Mitsuya K, Nakasu Y, Kurakane T, Hayashi N, Harada H, Nozaki K. Elevated preoperative neutrophil-tolymphocyte ratio as a predictor of worse survival after resection in patients with brain metastasis. J Neurosurg 2017;127(2):433-7.

27. Doi H, Nakamatsu K, Anami S, Fukuda K, Inada M, Tatebe H, et al. Neutrophil-to-Lymphocyte Ratio Predicts Survival After Whole-brain Radiotherapy in Non-small Cell Lung Cancer. In Vivo 2019;33(1):195201.

28. Nikolić I, Kukulj S, Samaržija M, Jeleč V, Žarak M, Orehovec B, et al. Neutrophil-to-lymphocyte and platelet-to-lymphocyte ratio help identify patients with lung cancer, but do not differentiate between lung cancer subtypes. Croat Med J 2016;57(3):287-92.

29. Li S, Lan X, Gao H, Li Z, Chen L, Wang W, et al. Systemic Inflammation Response Index (SIRI), cancer stem cells and survival of localised gastric adenocarcinoma after curative resection. J Cancer Res Clin Oncol. J Cancer Res Clin Oncol 2017;143(12):2455-68.

30. Proctor MJ, Morrison DS, Talwar D, Balmer SM, Fletcher CD, O'Reilly DS, et al. A comparison of inflammation-based prognostic scores in patients with cancer. A Glasgow Inflammation Outcome Study. Eur J Cancer 2011;47(17):2633-41. 